\title{
Fluorocarbon-peptideconjugates (FPC): new concept to increase the metabolic stability of peptides for therapeutic applications
}

\author{
Lucie Esteoulle $^{1}$, Adrien Flahault ${ }^{2}$, CendrineSeguin ${ }^{3}$, Sylvie Fournel ${ }^{1}$, Benot Frisch ${ }^{1}$, Xavier Iturrioz ${ }^{2}$, \\ Catherine Llorens-Cortes ${ }^{2}$, Dominique Bonnet ${ }^{3}$ \\ ${ }^{1}$ University of Strasbourg, France \\ ${ }^{2}$ INSERM - Collège de France, France \\ ${ }^{3} \mathrm{CNRS}$ - University of Strasbourg, France
}

https://doi.org/10.17952/35EPS.2018.265

\section{Introduction}

During the last decade, peptides have gained a wide range of applications in medicine. However, they are often not directly suitable for use as convenient therapeutics because they have intrinsic weaknesses, including poor chemical and physical stability, and short in vivo half-life.[1] To increase their metabolic stability, various strategies have been developed that all present some limitations. Among them, the acylation of peptides with hydrocarbon chains (H-chains) such as a palmitoyl moiety has been developed to extend their half-life in the blood circulation as reported for liraglutide.[2] However, H-chains increase also the association with cell membranes as well as the hemolytic activity and the potential toxicity of modified peptides, such as observed for antimicrobial lipopeptides. As a consequence, the development of an alternative approach to increase the metabolic stability of peptides is needed.

While the grafting of $\mathrm{H}$-chains onto peptides has been reported to enhance the circulation half-life of peptides, the incorporationof fluorocarbon chains (F-chains) within the sequence of potential therapeutic peptides has been sparsely described to date. $[3,4,5]$ However, F-chains have unique properties, very different from those of $\mathrm{H}$-chains. They are especially more stable, stiffer, lipophobic, and significantly more hydrophobic. In addition, F-chains are biologically inert molecules devoid of intrinsic pro-inflammatory, immuno-stimulatory activity or immunogenicity.[6] Considering those properties, we envisioned the grafting of F-chains onto peptides especially those targeting GPCRs that represent the target of more than $30 \%$ of drugs currently on the market. To test our novel approach called Fluorocarbon-Peptide Conjugation (FPC), the neuro-vasoactive peptide, apelin, previously reported for its low in vivo half-life (in the minute range) has been selected as a model. Apelin was isolated from bovine stomach extracts and identified as the natural ligand of the human orphan GPCR, APJ (ApelinR).[7] In the last decade, apelin and its receptors have emerged as playing a prominent role in controlling body fluid homeostasis and cardiovascular functions, with potential interesting therapeutic application in the treatment of cardiovascular diseases, water retention and/or hyponatremia. [8]

\section{Results and Discussion}

Herein, we describe the unprecedented FPC approach based on the grafting of F-chain onto apelin for improving its metabolic stability. To highlight the advantages of using F-chains over H-chains, fluoroapelin and lipoapelin were synthetized and their plasma stability, binding affinity for ApelinR, in vitro cytotoxicity and pro-inflammatory activities as well as their in vivo efficacy to decrease blood pressure in normotensive rats were carefully evaluated. Fluoroapelin were synthetized on solid-phase following a Fmoc/tBu approach. Based on the molecular modeling of the three-dimensional structure of the human ApelinR, we decided to incorporate a F-chain $\left(\mathrm{CF}_{3}\left(\mathrm{CF}_{2}\right)_{7}\left(\mathrm{CH}_{2}\right)_{2} \mathrm{CO}\right.$, F8-chain) on the $N$-terminal part of apelin. To highlight the advantages of using a F-chain over a H-chain, lipoapelin was obtained as control following the same approach.

The potential impact of F8-chain on the binding affinity of fluoroapelin for ApelinR was assessed by competition binding experiments using $\mathrm{CHO}$ cell membranes stably expressing the human ApelinR and $\left[{ }^{125} \mathrm{I}\right]-\mathrm{pE} 13 \mathrm{~F}$ as radioligand. Hence, as shown in Table 1, the affinity of fluoroapelin remained in the nanomolar range, similarly to that measured for apelin demonstrating the low impact of F8-chain on the binding properties of fluoroapelin. The plasma stability of fluoroapelin was then measured in the presence of different plasma species (mouse, rat and human)at $37{ }^{\circ} \mathrm{C}$ demonstrating that the grafting of F8-chain enabled to dramatically increase the half-life of apelin from few minutes to more than $24 \mathrm{~h}$. Similar results were obtained with lipoapelin (Table 1). 
Table 1: Structure, binding affinities and plasma stabilities of apelin, fluoroapelin and lipoapelin

$$
\mathbf{R}_{\mathbf{1}} \text {-KFRRQRPRLSHKGPMPF-OH }
$$

\begin{tabular}{cccc}
\hline Peptides & $\mathrm{R}^{1}$ group & $\begin{array}{c}\text { ApelinR binding, } \\
K_{\mathrm{i}}(\mathrm{nM})\end{array}$ & $\begin{array}{c}\text { Half-life in plasma } \\
(\mathrm{min})\end{array}$ \\
\hline Apelin & - & $0.060 \pm 0.010$ & $4.6 \pm 0.6$ \\
Lluoroapelin & & $0.210 \pm 0.027$ & $>1440$ \\
& & &
\end{tabular}

To highlight the advantages of using the FPC approach vs the classical lipidation approach, the cell toxicity of fluoroapelin and lipoapelin was evaluated on RAW 264.7 macrophages by MTS-based assay (Figure 1a). Interestingly, fluoroapelin, as well as apelin, did not show any significant increase of cytotoxicity for concentrations up to $30 \mu \mathrm{M}$ whereas lipoapelin displayed a high cytotoxicity even at low concentrations. In addition, lipoapelin exhibited higher hemolytic and pro-inflammatory effects than fluoroapelin (data not shown) demonstrating the higher biocompatibility of fluoropeptides as compared to lipopeptides. Finally, the efficacy of fluoroapelin was evaluated in vivo on arterial blood pressure (BP) in alert normotensive Sprague Dawley rats (Figure 1b). The intravenous injection of apelin $(15 \mathrm{nmol} / \mathrm{kg})$ did not significantly decrease arterial BP. In contrast, the intravenous injection of fluoroapelin and lipoapelin at the same dose $(15 \mathrm{nmol} / \mathrm{kg})$ maximally decreased arterial BP by $59 \pm 3$ and $38 \pm 2 \mathrm{mmHg}$, respectively. Thereby, fluoroapelin was found 2-fold and 16-fold more efficient at decreasing arterial BP than lipoapelin and apelin, respectively. The high efficiency of fluoroapelin to decrease arterial BP compared to apelin is due both to an increase in the intensity and duration of the hypotensive response.
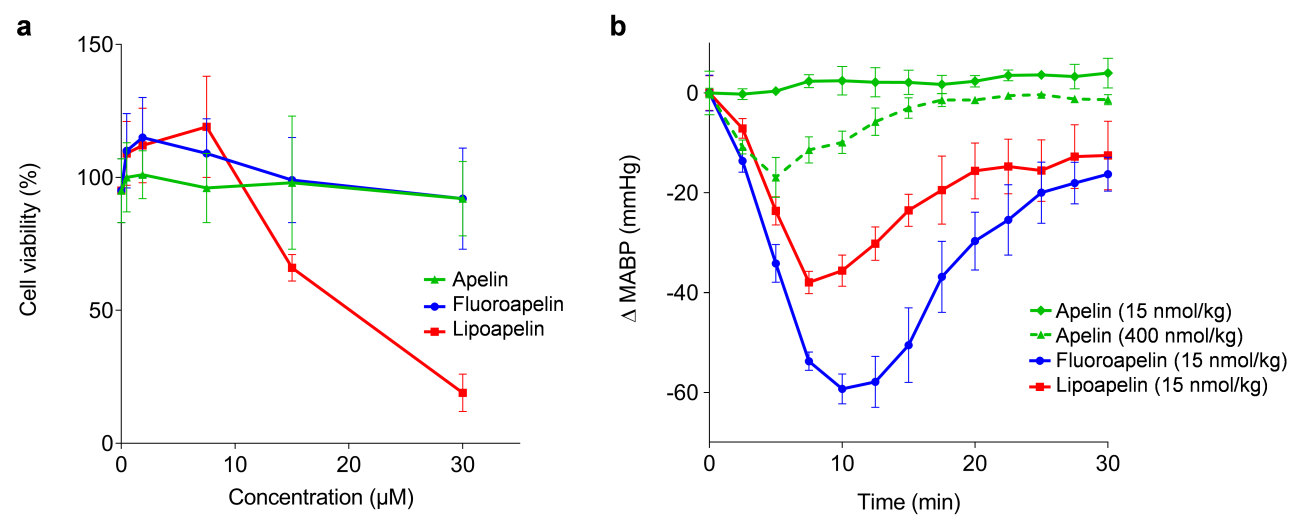

Figure 1: (a) Cytotoxicity and (b) in vivo effects on arterial blood pressure in alert normotensive rats of apelin, fluoroapelin and lipoapelin

\section{Conclusion}

As demonstrated for apelin, fluoropeptides are expected to decrease the hemolytic, cytotoxic and proinflammatory effects of lipopeptides and to improve their specificity of action both in vitro and in vivo. Compared to the classical lipidation of peptides, the FPC strategy offers therefore a more biocompatible, specific and efficient approach for enhancing the half-life of peptides and to facilitate their development as powerful pharmacological probes and/or drug candidates.

\section{Acknowledgments}

This work was supported by the Agence Nationale de la Recherche, the Centre National de la Recherche Scientifique, the Institut National de la Santé et de la Recherche Médicale (INSERM), the Université de Strasbourg and the Frontier Research in Chemistry (FRC). 


\section{References}

1. Vlieghe P., Lisowski V., Martinez J. Drug Discov. Today 2010, 15, 40-56.

2. Buse J.B., et al. Lancet 2009, 374, 39-47.

3. Francis J.N. et al. Vaccine 2015, 33, 1071-1076.

4. Malik L., et al. Langmuir 2012, 28, 593-603.

5. Gerbier R., et al. FASEB J. 2017, 31, 687-700.

6. Krafft M.P. and Riess J.G. Chem. Rev. 2009, 109, 1714-1792.

7. Tatemoto K., et al. Biochem. Biophys. Res. Commun. 1998, 251, 471-476.

8. O'Carroll A.M., Lolait S.J., Harris L.E. and Pope G.R. J. Endocrinol. 2013, 219, R13-R35. 